\title{
X-ray Phase Nanotomography through Ptychographic Coherent Lensless Imaging
}

\author{
Manuel Guizar-Sicairos, Ana Diaz, Andreas Menzel, and Oliver Bunk \\ Swiss Light Source, Paul Scherrer Institut, CH-5232 Villigen PSI, Switzerland
}

\begin{abstract}
We describe experimental and algorithmic developments of ptychographic X-ray computed tomography, a recently reported technique that enables three-dimensional, quantitative X-ray microscopy with high sensitivity. The technique is based on the incorporation of sample rotation and tomographic reconstruction to scanning X-ray diffraction microscopy (SXDM), a robust technique for two-dimensional X-ray coherent lensless imaging.
\end{abstract}

Keywords: X-ray tomography, phase retrieval, image reconstruction techniques, coherent diffractive imaging.

\section{INTRODUCTION}

For many applications in both biology and materials science, the examination of the three-dimensional structure of a sample with high resolution is highly desirable. X-ray tomography is for many cases an attractive technique for such studies as it allows three-dimensional imaging of structures within the sample in a non-destructive fashion. Preserving the sample for further studies can in some cases be of crucial importance, as often sample preparation is difficult, expensive, and time consuming.

There have been great advances in soft X-ray tomography for the study of cells with a resolution reaching $60 \mathrm{~nm} .{ }^{1}$ Soft X-ray imaging enjoys a high absorption contrast and high efficiency in the focusing and image forming optics, which results in high quality tomographic images that reveal, for example, the internal structure of cells with high resolution. ${ }^{1}$ This high absorption, however, also limits the penetration depth of soft X-rays, and their long wavelength limits the imaging depth of field (DOF), i.e. the axial range at which the image remains at focus. Both of the latter restrictions effectively constraint the sample thickness that can be imaged.

On the other side, hard X-rays have a significantly reduced absorption and increased DOF, making them more suitable to image larger and thicker samples. This capability is important to study tissue and cellular networks or, for the case of materials science, samples that are large enough to be considered a representative section of the bulk material. Despite its reduced absorption, the sample produces a phase shift on the incident $\mathrm{X}$-ray waves that can be used as a contrast mechanism for imaging. ${ }^{2-5}$

\section{PTYCHOGRAPHIC X-RAY COMPUTED TOMOGRAPHY}

Scanning X-ray diffraction microscopy (SXDM) is a well-established coherent lensless imaging technique that relies on phase retrieval image reconstruction from measurement of X-ray far-field diffraction patterns. ${ }^{6-9}$ In contrast to more conventional phase retrieval techniques that utilize a support constraint, ${ }^{10} \mathrm{SXDM}$ reconstruction algorithms exploit an overlap constraint. The illumination of the sample is confined to a small region using a pinhole or X-ray focusing optics, and diffraction patterns are measured with the sample translated to different positions in a direction perpendicular to the direction of propagation of the incident X-ray beam. A spatially finite illumination is necessary to ensure adequate sampling of the diffraction patterns. The illuminated regions of the sample have some degree of overlap which provides the phase retrieval algorithm with redundancy that allows fast, robust, and reliable reconstructions even for cases in which the incident illumination is not known. ${ }^{7-9}$ The resolution of SXDM is not determined by the size of the illumination or translations, but rather by the largest angle at which the diffraction pattern can be measured above the noise level. Because of the similarity with ptychography in the data acquisition procedure, ${ }^{11}$ SXDM is also referred to as ptychographic coherent diffractive imaging.

Contact information: manuel.guizar-sicairos@psi.ch

22nd Congress of the International Commission for Optics: Light for the Development of the World,

edited by Ramón Rodríguez-Vera, Rufino Díaz-Uribe, Proc. of SPIE Vol. 8011, 80118F

2011 SPIE · CCC code: $0277-786$ X/11/\$18 - doi: $10.1117 / 12.903688$

Proc. of SPIE Vol. $801180118 \mathrm{~F}-1$ 
The combination of SXDM with sample rotation to provide tomographic reconstructions was recently demonstrated. ${ }^{5}$ For a given sample orientation $\theta$, the SXDM reconstruction, $t(x, y, \theta)$, is related to the complex-valued index of refraction through a Radon transform, namely

$$
t(x, y, \theta)=\exp \left\{\frac{i 2 \pi}{\lambda} \int\left[n\left(\mathbf{r}^{\prime}\right)-1\right] \mathrm{d} z\right\},
$$

where $(x, y)$ are the transverse Cartesian coordinates and $z$ the direction of X-ray propagation, $\lambda$ is the wavelength of the illumination, $n(\mathbf{r})=1-\delta(\mathbf{r})+i \beta(\mathbf{r})$ is the three-dimensional complex-valued index of refraction and $\mathbf{r}^{\prime}=(x \cos \theta-z \sin \theta, y, z \cos \theta+x \sin \theta)$.

The phase of the reconstructions, $t(x, y, \theta)$, corresponds to the integrated phase shift of the X-ray beam as it traverses the sample. This integrated phase shift is proportional to the projection of the real part of the refractive index, which in turn is proportional to the sample electron density if the photon energy is sufficiently far from absorption edges. Because multiple waves of phase can be measured with SXDM the technique remains quantitative even for large phase shifts and non-negligible absorption. ${ }^{5,12}$ The latter properties makes the technique of high practical value for a wide range of samples.

Prior to the tomography step, further image processing is required on the reconstructed projections. Firstly, due to inherent ambiguities in SXDM reconstructions ${ }^{13}$ the phase may contain constant and linear additive terms that should be removed. Secondly, because of hardware limitations and sample drift, the reconstructed projections are misaligned. Lastly, the reconstructed phase is wrapped, thus only known modulo $2 \pi$, a limitation that has been dealt with in the past with a sometimes problematic step of phase unwrapping.

Since its original demonstration reported in Ref. 5, we have further developed automated algorithms to effectively deal with these limitations. In the following we provide a brief description of these methods and refer the reader to Ref. 13 for further details. We implemented an automatic approach for removal of the constant and linear additive terms which is based on minimizing the phase on regions known to be empty. This method is insensitive to phase wrapping discontinuities and approaches a root-mean-squared phase minimization in the limit of small noise in the phase reconstructions.

For alignment of the phase projections in the $x$-direction, which is perpendicular to the rotation axis, we align with respect to the center of mass of the sample. This is achieved using the fact that the center of mass of the projection gives the projected coordinate of the 3D center of mass. ${ }^{14}$ Because the sample is not isolated vertically, we do not measure its whole extent along the vertical $y$-direction, and we have found that the center of mass approach does not guide the algorithm to a correct vertical alignment. For alignment in the $y$-direction we instead use an approach based on registration of the sample vertical mass fluctuations. ${ }^{13}$ This approach has shown to be accurate and very robust and should be useful for alignment of projections from other tomography approaches, such as those based on direct imaging using optics.

We have additionally implemented a computed tomography algorithm that can work directly with wrapped phase, such that phase unwrapping can be altogether avoided for the tomographic step. ${ }^{13}$ We should note that the alignment procedure operates on an unwrapped portion of the projections, for this we choose a small and well-behaved portion of the projections where the unwrapping is easiest. These regions are straightforwardly identified by looking at the distribution of phase residues in the projection. ${ }^{15}$ Because the tomographic step is insensitive to wrapping, the imaging field-of-view is not constrained to the unwrapped region.

\section{CONCLUSIONS}

Ptychographic X-ray computed tomography delivers detailed quantitative 3D maps of electron density, and we routinely achieve a resolution in the range of $150 \mathrm{~nm}$. In our approach we use the phase of SXDM reconstructions to accurately retrieve the integrated phase along the X-ray path and exploit the high penetration and DOF of hard X-rays to measure samples of up to 70 microns thick.

We have developed improved algorithms for post-processing and alignment of projections that lead to improved image quality and take better advantage of the measurements. For example, with the modified computed tomography algorithm we can readily reconstruct regions of the sample where phase unwrapping is problematic. 
These new post-processing methods already take us a step further towards full automation of the tomographic procedure, and although some adjustments are still occasionally needed, it has reduced significantly the need for human supervision and interaction.

The versatility of this technique is already being applied for imaging cellular networks in human and animal bone, cellular plant tissue, hardened cement paste, and colloidal samples.

\section{ACKNOWLEDGMENTS}

We will present results of X-ray experiments performed on the cSAXS beamline at the Swiss Light Source, Paul Scherrer Institut, Switzerland. We also acknowledge samples, collaboration and/or fruitful discussions from M. S. Lucas, R. A. Wepf, P. Trtik, I. Chiapparini, P. Schneider, P. Thibault, M. Dierolf, C. M. Kewish, B. Enders, A. Fehringer, F. Marone, and M. Stampanoni. We thank X. Donath for his technical support.

\section{REFERENCES}

[1] C. A. Larabell and M. A. Le Gros, "X-ray tomography generates 3-D reconstructions of the yeast, Saccharomyces cerevisiae, at 60-nm resolution," Molecular Biology of the Cell 15, 957-962 (2004).

[2] P. Cloetens, W. Ludwig, J. Baruchel, D. Van Dyck, J. Van Landuyt, J. P Guigay, and M. Schlenker, "Holotomography: Quantitative phase tomography with micrometer resolution using hard synchrotron radiation x rays," Appl. Phys. Lett. 75, 2912-2914 (1999).

[3] F. Pfeiffer, O. Bunk, C. David, M. Bech, G. Le Duc, A. Bravin, and P. Cloetens, "High-resolution brain tumor visualization using three-dimensional x-ray phase contrast tomography," Phys. Med. Biol. 52, 69236930 (2007).

[4] M. Stampanoni, R. Mokso, F. Marone, J. Vila-Comamala, S. Gorelick, P. Trik, K. Jefimovs, and C. David, "Phase-contrast tomography at the nanoscale using hard x-rays," Phys. Rev. B 81, 140105 (2010).

[5] M. Dierolf, A. Menzel, P. Thibault, P. Schneider, C. M. Kewish, R. Wepf, O. Bunk, and F. Pfeiffer, "Ptychographic x-ray computed tomography at the nanoscale," Nature 467, 436-439 (2010).

[6] J. M. Rodenburg and H. M. L. Faulkner, "A phase retrieval algorithm for shifting illumination," Appl. Phys. Lett. 85, 4795-4797 (2004).

[7] M. Guizar-Sicairos and J. R. Fienup, "Phase retrieval with transverse translation diversity: a nonlinear optimization approach," Opt. Express 16, 7264-7278 (2008).

[8] P. Thibault, M. Dierolf, A. Menzel, O. Bunk, C. David, and F. Pfeiffer, "High-resolution scanning x-ray diffraction microscopy," Science 321, 379-382 (2008).

[9] A. M. Maiden and J. M. Rodenburg, "An improved ptychographical phase retrieval algorithm for diffractive imaging," Ultramicroscopy 109, 1256-1262 (2009).

[10] J. R. Fienup, "Phase retrieval algorithms: a comparison," Appl. Opt. 21, 2758-2769 (1982).

[11] B. C. McCallum and J. M. Rodenburg, "Two-dimensional demonstration of Wigner phase-retrieval microscopy in the STEM configuration," Ultramicroscopy 45, 371-380 (1992).

[12] A. Diaz, P. Trtik, M. Guizar-Sicairos, A. Menzel, M. Dierolf, P. Thibault, and O. Bunk, "Quantitative x-ray phase-contrast tomography with resolution in the $100 \mathrm{~nm}$ range," manuscript in preparation (2011).

[13] M. Guizar-Sicairos, A. Diaz, M. Holler, M. S. Lucas, A. Menzel, R. A. Wepf, and O. Bunk, "Phase tomography from x-ray coherent diffractive imaging projections," manuscript in preparation (2011).

[14] C.-C. Chen, J. Miao, and T. K. Lee, "Tomographic image alignment in three-dimensional coherent diffraction microscopy," Phys. Rev. B 79, 052102 (2009).

[15] R. M. Goldstein, H. A. Zebker, and C. L. Werner, "Satellite radar interferometry: Two-dimensional phase unwrapping," Radio Science 23, 713-720 (1988). 